\title{
Interactive Floor Support for Kinesthetic Interaction in Children Learning Environments
}

\author{
Kaj Grønbæk ${ }^{1}$, Ole Sejer Iversen ${ }^{2}$, Karen Johanne Kortbek ${ }^{3}$, \\ Kaspar Rosengreen Nielsen ${ }^{3}$, and Louise Aagaard ${ }^{4}$ \\ ${ }^{1}$ Department of Computer Science, University of Aarhus, \\ Åbogade 34, 8200 Århus N, Denmark \\ kgronbak@daimi.au.dk \\ ${ }^{2}$ Department of Information Science, \\ Helsingforsgade 14, 8200 Aarhus N, Denmark \\ imvoi@hum.au.dk \\ ${ }^{3}$ Alexandra Institute Ltd., Åbogade 34, 8200 Århus N, Denmark \\ \{kortbek, kaspar\} @alexandra.dk \\ ${ }^{4}$ Aarhus School of Architecture, Nørreport 20, 8000 Aarhus C, Denmark \\ louise.aagaardeaarch.dk
}

\begin{abstract}
This paper introduces a novel kinesthetic interaction technique for interactive floors. The interaction techniques utilize vision-based limb tracking on an interactive floor - a $12 \mathrm{~m}^{2}$ glass surface with bottom projection. The kinesthetic interaction technique has been developed for an interactive floor implemented in a school square. The paper discusses the kinesthetic interaction technique and its potentials in the domain of learning applications: Kinesthetic interaction supports body-kinesthetic learning as argued in the learning literature. Kinesthetic interaction is fun and motivating thus encourages children to explore and learn. Kinesthetic interaction on large display surfaces supports collaborative, co-located play and learning through communication and negotiation among the participants. Finally, the paper discusses prospects and challenges in development of kinesthetic interaction for interactive floors.
\end{abstract}

\section{Introduction}

The work reported was conducted in a project on interactive school environments. The objective was to create new types of IT-based learning experiences for school children. The work has been inspired from several sources namely empirical research in school environments [2,10], literature studies within multiple learning styles [5], and movement based interaction techniques $[13,18]$. Others have pointed to the fact that computer supported learning artifacts can stimulate learning through kinesthetic interaction. Underkoffler \& Ishii's [26] Illuminating Light workbench lets students learn about optical systems by designing them. In a use survey of the commercial Dance Dance Revolution ${ }^{1}$, Höysniemi [6] points to the fact that playing the kinesthetic interaction game has a positive effect on the social life and physical health of players, and improves endurance, muscle strength, and sense of rhythm by the users.

\footnotetext{
${ }^{1}$ www.konami.com
} 
A more thorough study of how bodies matter in interaction design is described in Klemmer et al. [12]. The empirical research in schools revealed challenges in moving it-support for multiple learning styles beyond the pure audio/visual styles and take advantage of kinesthetic learning styles. This challenge made us look into the field of interactive floors $[3,15,13,14,16]$.

Traditional computer games and learning-systems, running on PCs and laptops only challenge limited parts of the human body - usually only eyes, ears, and the pointing finger. But Gardner's [5] theory of multiple intelligences implies a need for multiple learning styles to provide optimal learning for a broader range of children.

Thus we developed an interactive floor platform for games and learning applications - it is called iGameFloor [6]. The platform has been implemented in Møllevangskolen school in Aarhus, Denmark, where is has got the nickname "Wisdom Well". However we denote the platform iGameFloor [6]. The iGameFloor is placed in a school department square where pupils spend both learning time and break time. It consists of a $12 \mathrm{~m}^{2}$ bottom projected interactive floor with vision based tracking of user movement. The work behind this paper has been carried out using this large scale installation for experimentation. A number of learning applications have been developed for iGameFloor.

iGameFloor supports three types of applications. Collaborative learning games. The kids work together through direct communication and kinesthetic interaction on the shared surface. The collaborative presence provides the social qualities of e.g. traditional board and card games. Knowledge sharing applications. A central placement of the iGameFloor in a department square makes it ideal for creating awareness of activities among classes. The floor is a remarkable [23] interface that draws attention to displayed information and encourage exploration. Simulations. The iGameFloor surface is ideal for simulating scenarios in a scale that immerses the kids beyond traditional computer displays. Learning about geometrics, physics, geography, and biology involves many examples of scenarios where there are learning potentials in simulations that may scale the content, simulating the users to be, e.g. nano particle size or stellar size.

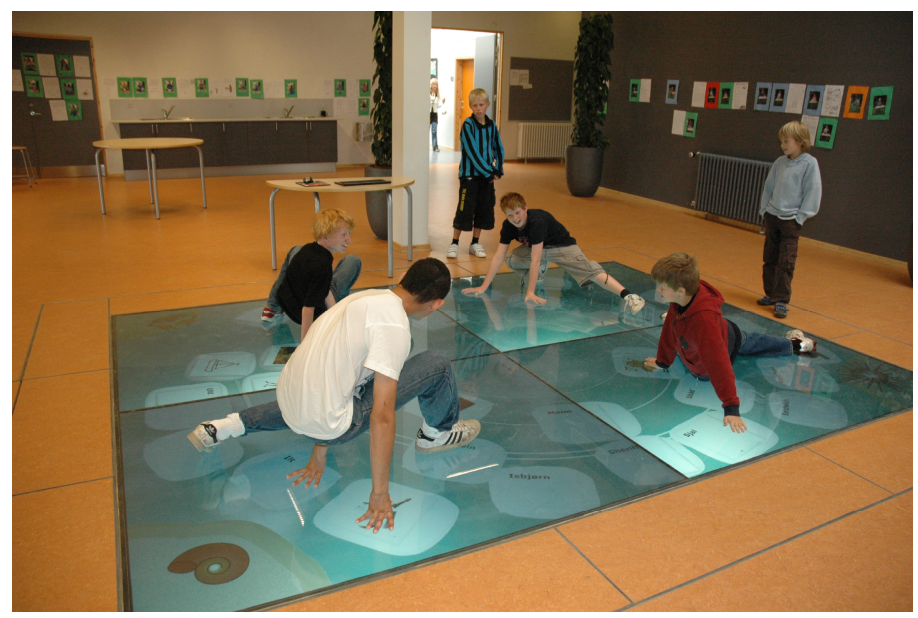

Fig. 1. iGameFloor in use at school 
The applications have been developed in a participatory design process [1,9] with children and teachers as participants. We particularly used the 'Fictional Inquiry' technique, $[2,9,10]$, which establishes a shared narrative design space between stakeholders in the design process; in this case among teachers, children and designers. This paper focus on the novel kinesthetic interaction techniques supported by iGameFloor.

\section{Interactive Floors}

Interactive floors with movement based interaction have emerged in recent years. They fall in two main categories: sensor-based and vision-based interactive floors.

Sensor-based interactive floors are typically utilized in dance and performances like set-ups, e.g. Magic Carpet [22] and Litefoot [3]. The prototypes are sensor intensive environments for tracking movements of feet. In Magic Carpet the sensor floor has been supplemented with sensor technologies for tracking the movements of the upper body and arms. To serve different shaping and sizes of an interactive floor the Z-tiles concept [16,24] was developed. As the above-mentioned systems the Z-tiles interactive floor is based on sensor technologies. Input from the sensors is used to control and manipulate sound providing the idea of playing an instrument with body movements. Another system exploring multi-user spatial interaction by means of a sensor based floor is the Virtual Space project [14]. The sensors are here used to enable spatial interaction and control of a computer game projected on a vertical positioned display. BodyGames [15] is a system consisting of tiles with touch sensors and light diodes supporting games where players invoke certain light patterns with their feet to gain points in the game. These sensor-based floors are typically limited to a discrete interaction with relatively large floor tiles. Finally, LightSpace ${ }^{\mathrm{TM}}$ (www.interactivefloor.com) is a commercial product based on tiles and sensors providing entertainment environments like dance floors

In contrast to the sensor-based floors, the vision based floors supports a more fluid and natural interaction on a floor surface. iFloor [13] introduces an interactive floor facilitating debate based on SMS and email contributions. A projector mounted on the ceiling is connected to a local computer to provide a display on the floor. The floor interaction works on the basis of a vision-based tracking package [27] analyzing the rim of the interface based on a video feed from a web-cam also mounted on the ceiling. The tracking of people's position and movement are interpreted as "magnetic" forces attracting a cursor with its home position at the center of the floor display. The force is proportional to the size of the shadow blob generated by a person moving under the projector. iFloor maintains precise tracking of up to 10 people at one time in a $4 * 5 \mathrm{~m}$ rectangle. People are tracked in a one meter band surrounding the display which is $3 * 4 \mathrm{~m}$ in size. A visual feedback is given in form of a projected string connecting the cursor and the user while being in the tracked area, thus people were made aware that they were taking part in the interaction. Finally, Natural Interaction ${ }^{2}$ has developed several prototypes of interactive floors based on tracking with vision technology.

\footnotetext{
${ }^{2}$ www.naturalinteraction.net
} 
The iGameFloor is built on the vision principles, but utilizing tracking of limb contact points from the bottom of the display. In the following, we will present the kinesthetic interaction technique in relation to children learning.

\section{Kinesthetic Interaction for Learning}

In recent years there has come focus on children and the need for more body movement. The tendency towards sedentary game playing in front of a TV or computer screen has generated a focus on lack of body movement and exercise. These gaming activities have been related to an increasing number of overweight children ${ }^{3}$. One way to address this problem is to combine body movement and the attractive digital game elements, as seen e.g. in EyeToy ${ }^{\mathrm{TM}}$ from Playstation2 ${ }^{\mathrm{TM}}$, Dance Dance Revolution and Nintendo Wii ${ }^{\mathrm{TM}}$.

Body movement is a central learning area for children [5], but the body is not limited to being a basis for movement activities or an instrument to impact on the surrounding environment. Body and movement stimulate the sensory system, which gives the individual experience and knowledge of her/his own identity as well as the physical and social outside world. This sense perception makes a basis for reflection and abstraction this way body movement also relates to cognition. The role and significance of the body movement in children's learning processes is crucial, especially in the pre-school age, not only concerning health and exercise questions, but also sensuality, aesthetic, social and personal learning. Children sense and learn through the body. Merleau-Ponty's phenomenology [17] of the body gives the body an essential significance in relation to the individual's recognition of her/himself and others.

Within the HCI community the discussion of human-computer interaction has primarily been discussed from a cognitive concern. With the rapid penetration of technology into everyday life and the following "aesthetic turn" in HCI [25] a concern for the human body as a locus of sensory-aesthetic appreciation is gaining acceptance. Moen [18] provides an account of design aspects of human movement when used as interaction modality between people and technology. She calls this modality for kinesthetic interaction. An examples is, the BodyBug, being a way to experience and interact with technology by use of the full faculty of the human body [19].

A popular kinesthetic interaction based game is the commercially available Nintendo Wii that utilizes accelerometers and gyroscopes in measuring user movements and transforming them to tennis or golf swings. Another example is the EyeToyPlay game (http://www.eyetoyplay.com/) for Playstation $2^{\mathrm{TM}}$. It is a TV game platform utilizing web camera input. The time-out based selection technique introduced by EyeToyPlay is also well-suited for vision based interactive floors, see section 4 .

The kinesthetic interaction approach is especially interesting when it comes to IT support in learning environments. Gardner [5] provides a theory of multiple intelligences emphasizing that each individual child possesses a unique combination of relatively autonomous intelligences including a body-kinesthetic intelligence. Traditional class-room teaching emphasizes the development of logical intelligence and linguistic intelligence excluding body-kinesthetic learning to the gyms.

${ }^{3}$ E.g. http://www.msnbc.msn.com/id/7722888/ 
According to Moser [20], educational research confirms that movement based playing can be relevant in developing social skills, and in this way social skills attached to body and movement may lead to improvement of speech. The iGameFloor sets a stage for this kind of activities, whether it is concrete sound/speech training according to a bottom-up method or a dialogue in "free" games according to a topdown method. The iGameFloor is a motivating place to train vocabulary through games - the Stepstone application being described in Section 6 is an example of this kind of training.

\section{4 iGameFloor Infrastructure}

In this section we briefly describe the infrastructure, a more extensive description can be found in [6]. The iGameFloor is built into the physical floor of a school department square. The iGameFloor is a $3 \mathrm{~m}$ deep hole covered with a projection surface. The projection surface is $3 * 4 \mathrm{~m}$ glass of approximately $9 \mathrm{~cm}$ thickness divided into four tiles. The glass surface consists of carrying glass, diffusion layer, and a hard protection surface glass. The four tiles are supported at the outer edges and with an internal conic frame resting on a supporting pillar in the center (see Fig. 2). The projection is created by 4 projectors with a resolution of 1024*768 pixels. They are placed vertically covering each their tile of glass. The projectors are driven by a PC with a graphics board with 4 DVI outputs. Each projector is associated with a Web cam tracking limb contact points on the tile covered by the given projector.

The four Web cams associated with the projectors are managed by a separate PC running the vision software supporting fine-grained tracking of limb positions. The limb positions are communicated to the application PC feeding the four projectors. The tracking PC can be switched to a mode, where it uses a ceiling mounted wideangel Creative webcam for coarse-grained tracking of body contours from above.

The iGameFloor supports sound through ceiling mounted loudspeakers and a subwoofer placed nearby the iGameFloor. The start up/shut down of computers and
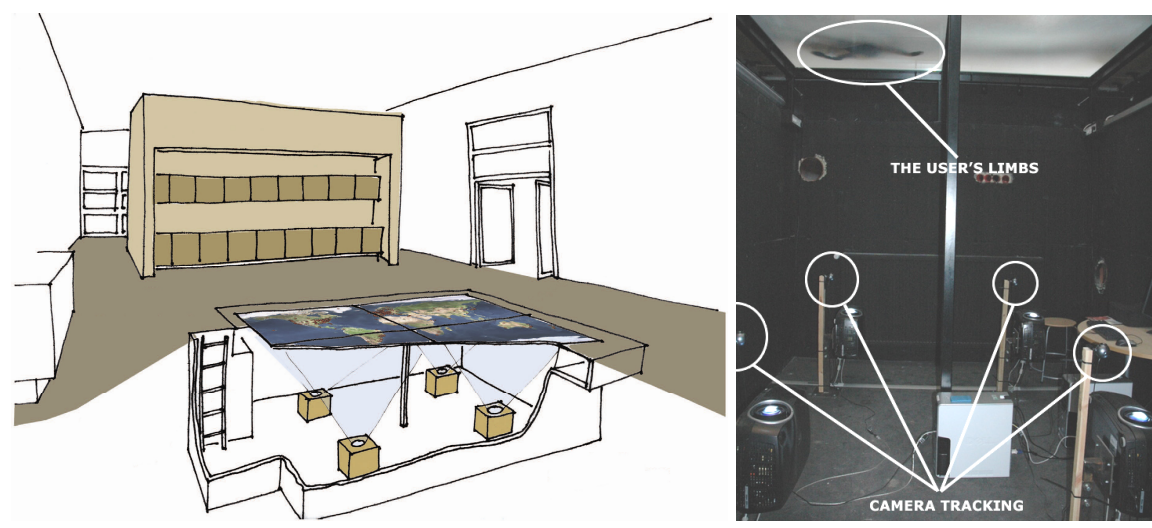

Fig. 2. Sketch and picture of the iGameFloor beneath the surface 
projectors is handled by a Creston control panel on a nearby wall. The panel also controls lighting and curtains. Having powered up the iGameFloor from the Creston panel, it can be fully controlled by body movement on the surface. A traditional keyboard and mouse is located on a nearby shelf to be used for editing purposes.

The basic software architecture is described in detail in [6], it consists of three layers: Server layer, client layer and sensor layer. The server layer consists of a web-server with a database. The database holds data and settings for the learning game applications and more general objects that can be referenced across applications. E.g. when a game is created in an application the user can specify type and the level of difficulty for the children. The client layer consists of a common Flash plus .NET framework for a number of learning game applications. This way Flash can tap into the power of .Net for storage connectivity, user interface widgets and operating system integration while maintaining the power of Flash for 2D game development. The general selection principle used is time-out based, i.e. sticking to a selection for a few seconds starts the invocation of an action. A Dashboard for switching applications and handling settings has been developed, in addition to the range of awareness, gaming and simulation applications. The sensor layer consists of four basement mounted cameras. The cameras track the location of the users and send the location to the application running on the floor. The basement mounted cameras also track the location of the users but does so by tracking the silhouette of the user's limbs through the glass plates on the floor. This will be described in greater detail in the following section.

Data from the cameras is sent to a tracking machine in the client layer that processes the data and applies an algorithm for assigning and maintaining identifiers for limb contact points between frames for use in the applications running on the floor. The algorithm compares the previous frame with the current frame of the camera input and if the two tracked limbs are within a specified number of pixels from each other they are most likely the same limb and will thus be assigned the same identifier between frames. Otherwise the limb will be assigned a new unique identifier.

\section{Kinesthetic Interaction Based on Limb Tracking}

One of the major innovations in the setup is the use of the basement mounted cameras for tracking the user's limbs like hands and feet. Positions and identifiers of limb contact points are used as input to the applications running on the floor. A ceiling mounted camera approach, as known from the iFloor project [13], is well suited for moving along a ribbon around the projection but it is not suitable when trying to track the position of the user in order to, e.g. activate a "button" on the floor. Another problem with the ceiling tracking approach is that users are seen in perspective and not directly from above making it hard to separate user's contours from each other. Moreover, it is hard to calculate precise positions to use for the individual users since the contour "blob" is quite big compared to the users contact points with the surface.

In contrast, the limb-based interaction technique offers a solution to these problems even though it is not as light-weight as the ceiling mounted approach. One of the main advantages in the approach is the elimination of the perspective problem since the user's limbs are in direct contact with the glass plates and only limbs in contact with the glass surface are tracked. The limbs can be tracked with a webcam since the 
contact points with the surface create a sufficient contrast from both the projection and the rest of the body under suitable light conditions, see Fig. 3. Limb positions are piped via TCP/IP to the applications. It is thus possible to hit a button in an application even though other users are standing close. Since only the center and not the contour of the limbs are being tracked the users are much less likely to disturb other users while interacting with the system. Fig. 3 shows a user's feet being tracked on the floor. The black circles indicate the center position of the user in the system and are very accurate compared to the physical location of the user.

\section{Conditions for Reliable Real-Time Limb Tracking}

There are some demands that need to be fulfilled for the system to track the user's limbs in a reliable way. One demand is a calibration in the tracking software based on various factors in the environment like ambient light and shadows. Another demand is that the light from above the iGameFloor must be strong enough to reduce the light emitted from the projectors in order to give the required contrast on the user's limbs when standing on the glass surface. Tests show that at least 300 lumen ambient light are required to obtain the desired contrast for tracking. Otherwise the dark areas in the projected interface will be tracked as input to the applications. This means the iGameFloor must be put in a controlled environment where the light on the iGameFloor is adjusted according to changes in the environment. To achieve this we use indirect light on the floor since the reflection from direct light can be disturbing for the users.

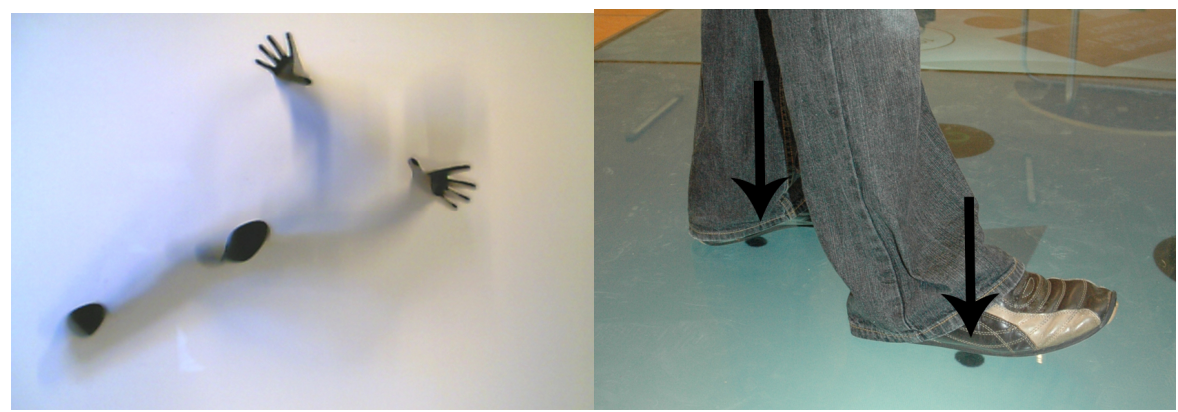

Fig. 3. Limb contact with the surface is tracked. The black dots to the right show the estimated positions of the users' limbs that are used in the applications.

When the system has been calibrated and adjusted to the light settings in the environment it is very robust and is able to track multiple users accurately. Tests have shown that up to fifteen users, i.e. at least 30 limb contact points can be tracked simultaneously on the floor though space is limited.

There is, however one limitation, to be aware of when designing the game play for applications on the iGameFloor. The problem may occur when the user places limbs directly on the reflection from the projector lens in the glass. This way the silhouette of the user's limb will be lighted up and can hardly be tracked by the system. Thus there are four dead spots in the current setup - one for each projector. 


\section{Applications Utilizing the Kinesthetic Interaction Technique}

In this section a brief introduction to some of the applications developed or redesigned for the iGameFloor is provided. The applications were developed as part of our research in limb-based tracking and kinesthetic interaction.

\section{Pong}

One of the first games implemented on iGameFloor was Pong - the classic videogame. Pong is a tennis-like game, and it calls for kinesthetic interaction in that the players may use their body movement to move the bat from side to side similar to real tennis or ping pong. On iGameFloor, it can be played by two players - one on each side of the iGameFloor and the side wards movements of their feet are tracked. The system keeps track of the players' scores and the player who first scores ten points has won the game. This game can be started by the children in the school breaks.

\section{iFloorQuest}

iFloorQuest is a floor game for four players inspired by Trivial Pursuit ${ }^{\mathrm{TM}}$. During the game a series of questions are "thrown at" the individual players from the center of the floor. Players then have a few seconds to jump to the one of the two spot with the possible answers. If a correct answer is selected both the player and the team will score a point since there is both an individual and collaborative score. In this way players can both play with or against each other. In most cases the children get very engaged and try to help each others in answering either through communication or by explicit jumping to the right answer - running the risk of missing ones own next question. As shown in Fig. 4, children sometimes team up 2 in each corner holding on to each other to make joint jumps to the answer spots. This way kinesthetic interaction has introduced engaging physical activity on top of a traditional sit down quest game.

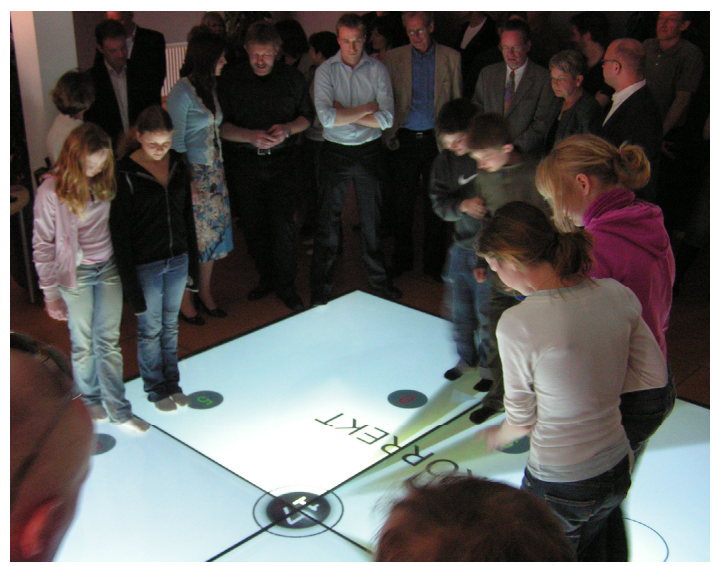

Fig. 4. iFloorQuest in use with 8 players 


\section{Stepstone}

Stepstone is a learning game application for up to four players. During the game a series of exercises are issued in speech and text, and the children must quickly use their hands and feet to combine a selection of several game elements to provide the correct solution. Stepstone functions as a framework in which different learning content can be implemented as required (e.g. the multiplication table, mapping language concepts to broader language concepts etc.), as well as the graphical environment can be varied (e.g. sea, rainforest, the city, the universe etc.). In this specific scenario, the setting is a beach with four shores in the corners and in the middle a sea filled with piranhas and stones for players to step on. Exercises require each player to select a combination of stones to provide a solution. The only way to avoid getting bitten is not to step in the water - or to solve the exercises correctly.

The objective in the game is to score as many points as possible by mapping pictures of concepts to categories over a specified amount of rounds. The players must select the correct answers by placing their hands or feet on the stones within the given time limit (visualized by movements in the water). Since the amount of correct answers exceeds the number of players, the players will have to use both hands and feet in order to score as many points as possible. When the time is up the selected answers will be evaluated and the player's collaborative score will be updated.

The interaction in the Stepstone application depends solely on kinesthetic interaction. Inspired by Twister released in 1966 by Milton Bradley Company, Stepstone uses body movement in a collaborative game frame. Whereas Twister is an edutainment game, Stepstone combines collaborative solving of learning exercises with the entertaining and engaging aspects of kinesthetic interaction. A variant of Stepstone was developed for hearing impaired children [11].
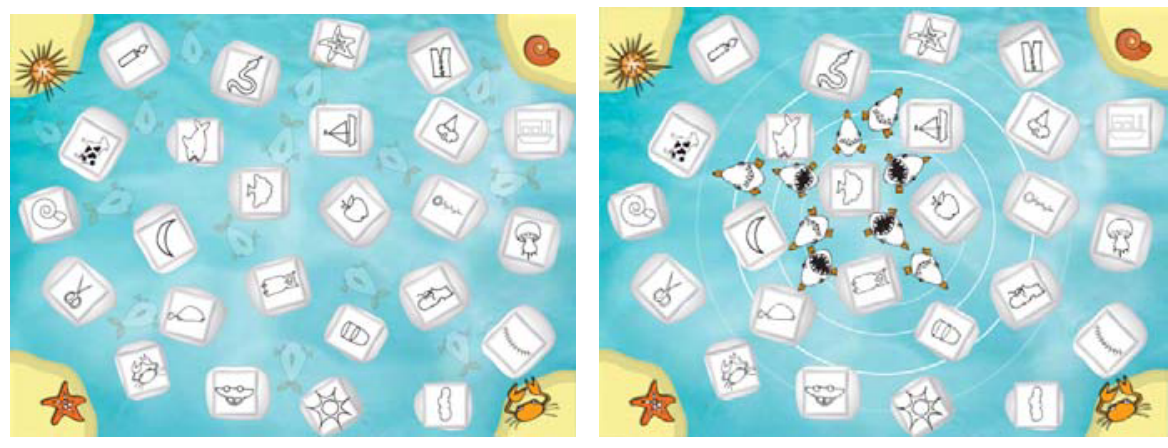

Fig. 5. Various stages in a Stepstone application

\section{Dashboard}

To avoid use of mouse and keyboard for controlling iGameFloor applications, a socalled dashboard has been developed. It is capable of performing standard task like switching between applications and basic control settings of the applications.

Since the dashboard has to function as a layer on top of the other iGameFloor applications, it is activated by a rare sequence of movements. The user walks from one 


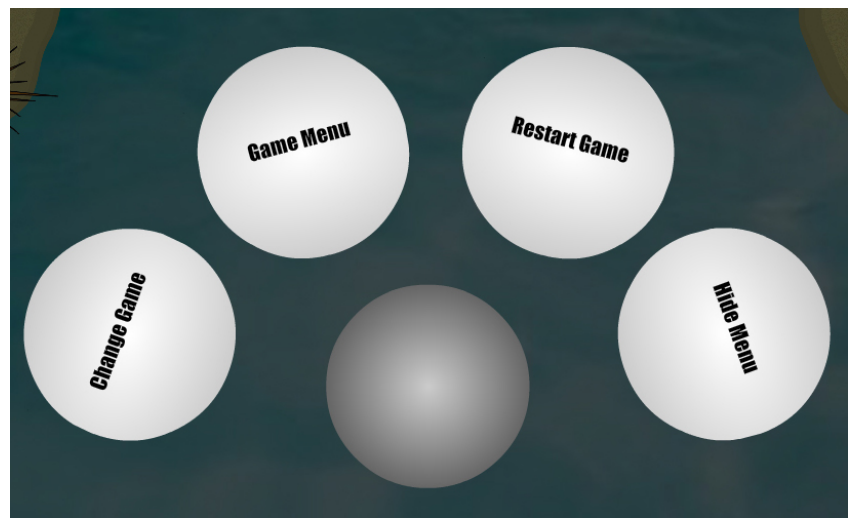

Fig. 6. Screen dump of the dashboard for application management

side of the floor to the other side and ends the sequence at the floor center. Here the Dashboard pops up in front of the user standing in the middle of the floor. It is a half pie variant of pie menus [8] that folds out in front of the user who can now select the action to take. Available options are e.g.: "Restart Game", "Hide Menu", "Game Menu" and "Change Game". If the "Change Game" option is triggered another half pie menu will fold out with available learning applications.

\section{Kinesthetic Interaction Lessons}

In the following, we describe qualitative lessons learned from the research and development phase. During the design project, participant observations of children's interacting on the iGameFloor were undertaken. Preliminary analysis of the observations brought about some general lessons regarding kinesthetic interaction and it's relation to learning environments.

\section{Achievements and Successes}

Full control possible through Kinesthetic Interaction. The iGameFloor can be fully operated using body movement. Children are able to interact with the iGameFloor using body movement as the only mean of interaction. When playing the iFloorQuest Application, children answered the questions by moving their body to the position of the right answer. The kinesthetic interaction was initiated without further instructions. When playing the Stepstone application, children immediately grasped Stepstone's metaphor and started stepping on the stones. Selections (corresponding to click and double click) are implemented by timeout triggers, i.e. standing still for 3 seconds may invoke a selection. Standing still for another 3 seconds may invoke an operation on the selection. Finally, generic functionality in the Dashboard can be invoked by a little rare movement sequence as described in Section 6; this kinesthetic sequence corresponds to pushing the Windows button on a PC or invoking the Dock on a Mac. One operation which we found hard to make kinesthetic is entry of data similar to keyboard entry. Thus a wireless keyboard is available close to the floor. 
Kinesthetic interaction stimulates collaborative learning. Children perceive the iGameFloor as a collaborative learning environment. During the games, children communicate with other participants and with the spectators in order to find the right solutions to the given game challenges. In the iFloorQuest as well as in Stepstone, the children treated each other as teammates on a team assignment; Exercises and challenges were discussed during the games with other team members and with spectators and strategic agreements were quickly negotiated in the teams with respect to prior experiences on the floor. Engaging shouting of proposals across the floor is often observed, and individual scores were less important than collective scores.

Kinesthetic interaction is fun and motivating. Children were generally engaged by the use of kinesthetic interaction and described the learning games as 'fun', 'motivating', for instance they were eager to make their own content for the games. When doing an assignment in geography a group of six graders themselves made 50 questions to the iFloorQuest application on geography. Then their classmates were invited to a 'battle' on the iGameFloor; A battle of capitals and famous sights worldwide. This particular iFloorQuest game was played with 8 participants and the rest of the class cheering or advising the participants in the geographical questions. Afterwards both children and the teacher found the activity rewarding.

Limb-tracking is more precise than contour tracking. Both iFloor [13] and EyeToyPlay interpret the contour of the users' shadows and use that as the "cursor" representing the user. In iFloor this makes it quite hard to conduct precise selections since the cursor position is set to the center of the shadow blob. In EyeToyPlay, it is the edge of the shadow blob that constitute the cursor, this makes it possible to cheat, e.g. in games expecting the player to jump, you may just wave your hand over your head, since a jump is detected as a generating a higher shadow. In contrast limb contact points are pretty small and assigning a cursor to the center or the edge of a limb contact point will both be precise and easy to interpret in relation to ones body.

\section{Limitations and Challenges}

Hard to identify individual players. A central issue for the game play is identification of individual players. Identifiers are relevant if the system was to keep track of the individual user's score. But it is hard to identify individuals solely based on shadows generated on a glass surface. For the first version of iGameFloor, we have made collaborative gaming applications that get around the identification issue either by associating a location to a specific player or to make the game a complete collaborative endeavor for a group of players to solve a common mission. In iFloorQuest, identification is handled by giving all users a base to stand on to not move freely around on all of the iGameFloor. In the Pong game, each end of the court is associated with a player. In the Stepstone learning game users can select stones all over the floor making it hard to track the score of a given user. Thus the game play was made collaborative for the group by calculating a common score. A possible future solution may be to identify users with different LED-light colors similar to MultiLightTracker [21]. If users interact with both hands and feet this will involve marking up both hands and feet with the same unique color. Another approach could be shape or visual tag tracking.

Children expecting a touch-sensitive surface. Some children assumed that they could interact with the iGameFloor using their feet as point and click/drag and drop. iGameFloor has some similarities with well-known interfaces from the familiar PC, 
thus it was quite common that children started stamping on icons and the like, expecting the computer to respond to kinesthetic clicks. In other incidences children double stamped on the iGameFloor during game as an attempt to generate a response by the computer as if they were double clicking a mouse. These observations all points to the need for a conceptual framework and standard audio/visual cues for kinesthetic interaction such that the interface doesn't lead to wrong expectations.

Missing the third dimension. Since the limb-based tracking is only 2D, we lack the possibility of taking advantage of users' movement in the third (vertical) dimension. Children expected iGameFloor to "be aware" of their hand gestures in the air. During a game session two 10 years old were moving their body as if the computer could track their body movement away from the floor. They wanted to be able to control a virtual ball on the floor by moving their upper body. To address this issue, we consider combining the limb-tracking with other sensors, e.g. taking advantage of the capabilities of children cell phones, e.g. Nokia 5500 and some Samsung models includes accelerometers and Bluetooth communication, thus it might be able to let the cell phone send signals about movements above the surface to iGameFloor. Another possibility might be to utilize a camera from the side tracking in the third dimension.

\section{Comparison to Related Work}

BodyGames [15] and LightSpace ${ }^{\mathrm{TM}}$ (www.interactivefloor.com) are two sensor and tile based floors with abstract light diode feedback. Compared to BodyGames and LightSpace $^{\mathrm{TM}}$ the iGameFloor provides a much richer visual multimedia feedback with extensive guidance on the activities undertaken.

Compared to the vision based iFloor system [13], the iGameFloor supports tracking of multiple users directly on the projection surface. iFloor only facilitated tracking on the edge of the floor. The iGameFloor also reduces the problems of shadowing the projection since the projection comes from beneath the users' bodies and do not disturb the projection except from what they hide with their feet on the surface. The iGameFloor also provides a much more fine-grained and precise tracking of users. The tracking is targeted to the contact points of limbs and not just the contour of the entire body as was the case in iFloor.

\section{Conclusion and Future Work}

This paper has introduced a novel kinesthetic interaction technique for a large scale interactive floor installation. The kinesthetic interaction technique utilizes visionbased limb tracking from the bottom of the interactive floor. Four cameras provide fine-grained tracking of limb (e.g. foot, hand, knee, and elbow) contact points. The setup allow real-time tracking of $30+$ limb contact point, thus providing large scale multi-user interaction in an interactive space. The kinesthetic interaction technique has been utilized to develop learning applications including games for school environments. It has been illustrated how the kinesthetic interaction technique can support real-time co-located collaborative learning games with many participants. Several new applications of the kinesthetic interaction technique is under development, and 
applications are not limited to school environments - experience environments like museums, attractions, and playgrounds or shopping malls are among the new application domains.

We thus see many possible extensions to the concept. We could e.g. start detecting difference between hands and feet and we may be able to track different foot gestures or patterns that could be used to invoke different actions in the applications.

We also wish to investigate how limb-tracking may be supported without the expensive bottom tracking of floors, since many of the applications may be executed on a much cheaper platform with projection from above or in some future with new "electronic paper" based display put on top of floors.

A structured evaluation of the kinesthetic interaction and learning potentials on specific educational tasks will take place when the system is finally released at the school.

\section{Acknowledgements}

This work was supported by ISIS Katrinebjerg, Center for Interactive Spaces. We wish to thank all our center colleagues as well as the staff at Department of Education, Aarhus Municipality and Møllevangskolen for contributions to the work. We would also like to thank the sponsors of the physical installation: the Oticon Foundation, Boligfonden Kuben, NNC, Arkitema, Søren Jensen Engineering, and Dansk Data Display.

\section{References}

1. Bødker, S., Grønbæk, K., Kyng, M.: Cooperative Design: Techniques and Experiences from the Scandinavian Scene. In: Baecker, et al. (eds.) Readings in Human- Computer Interaction: Toward the Year 2000, Morgan Kaufman Publishers, San Francisco, USA (1995)

2. Dindler, C., Eriksson, E., Iversen, O.S., Lykke-Olesen, A., Ludvigsen, M.: Mission from Mars: a method for exploring user requirements for children in a narrative space. In: Proceeding of the 2005 Conference on interaction Design and Children. IDC '05, Boulder, Colorado, June 08 - 10, 2005, pp. 40-47. ACM Press, New York (2005)

3. Fernström, M., Griffith, N.: Litefoot - Auditory Display of Footwork. In: Proceeding of ICAD'98, Glasgow, Scotland (1998)

4. Fogarty, J., Forlizzi, J., Hudson, S.E.: Aesthetic Information Collages: Generating Decorative Displays that Contain Information. In: Proceedings of UIST'01, pp. 141-150. ACM Press, New York (2001)

5. Gardner, H.: Frames of Mind: The theory of multiple intelligences. In: Britain by Fontana Press, 2nd edn., p. 466. Basic Books, New York (1993)

6. Grønbæk, K., Iversen, O.S., Kortbek, K.J., Nielsen, K.R., Aagaard, L.: iGameFloor - a Platform for Co-Located Collaborative Games. In: Proceedings of the International Conference on Advances in Computer Entertainment 2007, Salzburg, Austria (June 13-15, 2007)

7. Höysniemi, J.: International survey on the Dance Dance Revolution game. ACM Computers in Entertainment 4(2) (2006) 
8. Hopkins, D.: The Design and Implementation of Pie Menus. in Dr. Dobb's Journal (1991)

9. Iversen, O.S., Brodersen, C.: Bridging the Gap between users and children - A sociocultural approach to designing with children in Springer's journal Cognition. Technology and Work for the special issue on Child-Computer Interaction: Methodological Research 9(2) (2007)

10. Iversen, O.S.: Participatory Design beyond Work Practices - Designing with Children, Ph.D. Thesis, Dept. of Computer Science, University of Aarhus (2006)

11. Iversen, O.S., Kortbek, K.J., Nielsen, K.R., Aagaard, L.: Stepstone- An Interactive Floor Application for Hearing Impaired Children with a Cochlear Implant. In: Proc. of IDC07 6th International Conference on Interaction Design and Children, Aalborg, Denmark (June 6-8, 2007)

12. Klemmer, S.R., Hartmann, B., Takayama, L.: How bodies matter: five themes for interaction design. In: Proc. of the 6th ACM Conference on Designing interactive Systems. DIS '06, University Park, PA, USA, June 26-28, 2006, pp. 140-149. ACM Press, New York (2006)

13. Krogh, P.G., Ludvigsen, M., Lykke-Olesen, A.: Help me pull that cursor - A Collaborative Interactive Floor Enhancing Community Interaction. In: Proc. of OZCHI, University of Wollongong, Australia. CD-ROM, 22-24 November, 2004 (2004) ISBN:1 74128079

14. Leikas, J., Väätänen, A., Räty, V.: Virtual space computer games with a floor sensor control: human centred approach in the design process. In: Brewster, S., Murray-Smith, R. (eds.) HH-CI. LNCS, vol. 2058, pp. 199-204. Springer, Heidelberg (2001)

15. Lund, H.H., Klitbo, T., Jessen, C.: Playware Technology for Physically Activating Play. Artificial life and Robotics Journal 9 (2005)

16. McElliot, L., Dillon, M., Leydon, K., Richardson, B., Fernstrom, M., Paradiso, J.: ForSe FIElds - Force Fields for Interactive Environments. In: Proc. of Ubiquitous Computing. 4th Int. Conference, Göteborg Sweden, pp. 168-175 (2002)

17. Merleau-Ponty, M.: Phenomenology of Perception translated by Colin Smith. Humanities Press, New York (1945)

18. Moen, J.: KinAesthetic Movement Interaction: Designing for the Pleasure of Motion, dissertation from KTH, Numerical Analysis and Computer Science, Stokholm, Sweden (2006)

19. Moen, J., Sandsjö, J.: BodyBug - Design of KinAesthetic Interaction. In: Digital Proceedings of NORDES In the Making, Copenhagen, Denmark (2005)

20. Moser, T.: Bevægelse i sproget - sproget i bevægelse (in Danish) http://kidlld.dk/temaer/ krop/beveagelse/ PloneArticle_view

21. Nielsen, J., Grønbæk, K.: MultiLightTracker: Vision based simultaneous multi object tracking on semi-transparent surfaces. In: Proc. of the International Conference on Computer Vision Theory and Applications (VISAPP 2006), Setúbal, Portugal, 25-28 February, 2006 (2006)

22. Paradiso, J., Abler, C., Hsiao, K., Reynolds, M.: The Magic Carpet - Physical Sensing for Immersive Environments. In: Proc. of CHI' 97, Atlanta, GA, USA (1997)

23. Petersen, M.G.: Remarkable computing: the challenge of designing for the home. In: Proceedings of CHI'2004, pp. 1445-1449. ACM Press, New York (2004)

24. Richardson, B., Leydon, K., Fernstrom, M., Paradiso, J.A.: Z-Tiles: building blocks for modular, pressure-sensing floorspaces April 2004 Extended abstracts of the 2004 conference on Human factors and computing systems (2004)

25. Udsen, L.E., Jørgensen, A.H.: The aesthetic turn: unraveling recent aesthetic approaches to human-computer interaction. In Digital Creativity 16(4), 205-216 (2005) 
26. Underkoffler, J., Ishii, H.: Illuminating light: An optical design tool with a luminoustangible interface. In: Proc. of the SIGCHI conference on Human factors in computing systems, ACM Press, Los Angeles (1998)

27. Valli, A.: RETINA - video tracking software available at (2004-06-18), http://alessandrovalli.com/retina/ 